Cahiers $d u$ MONDE RUSSE

\section{Cahiers du monde russe}

Russie - Empire russe - Union soviétique et États indépendants

$62 / 4 \mid 2021$

Varia

\title{
Jonathan DALY, éd., Pillars of the Profession. The Correspondence of Richard Pipes and Marc Raeff
}

\section{Andreï Kozovoï}

\section{(2) OpenEdition}

\section{Journals}

Édition électronique

URL : https://journals.openedition.org/monderusse/12995

DOI : 10.4000/monderusse. 12995

ISSN : $1777-5388$

\section{Éditeur}

Éditions de l'EHESS

\section{Édition imprimée}

Date de publication : 1 décembre 2021

Pagination : 775-780

ISBN : 978-2-7132-2895-7

ISSN : $1252-6576$

\section{Référence électronique}

Andreï Kozovoï, « Jonathan DALY, éd., Pillars of the Profession. The Correspondence of Richard Pipes and Marc Raeff », Cahiers du monde russe [En ligne], 62/4 | 2021, mis en ligne le 01 décembre 2021, consulté le 03 septembre 2022. URL : http://journals.openedition.org/monderusse/12995 ; DOI https://doi.org/10.4000/monderusse. 12995

Ce document a été généré automatiquement le 3 septembre 2022.

Tous droits réservés 


\title{
Jonathan DALY, éd., Pillars of the Profession. The Correspondence of Richard Pipes and Marc Raeff
}

\author{
Andreï Kozovoï
}

\section{RÉFÉRENCE}

Jonathan DALY, éd., Pillars of the Profession. The Correspondence of Richard Pipes and Marc

Raeff, Leiden - Boston : Brill (Eurasian Studies Library), 2019, 433 p.

1 La correspondance entre historiens a donné lieu à plusieurs chefs-d'œuvre du genre épistolaire: on songe à l'échange lumineux entre les pères fondateurs de l'école des Annales, Marc Bloch et Lucien Febvre, ou celle, transfrontalière et polémique, entre François Furet et Ernst Nolte ${ }^{1}$. Jonathan Daly, professeur à l'université de l'Illinois à Chicago et spécialiste de la police tsariste ${ }^{2}$, ancien doctorant de Richard Pipes qui a hérité de ses archives personnelles en vue d'une biographie ${ }^{3}$, nous livre ici une édition critique, dans le meilleur sens de ce mot, de la correspondance entre deux «piliers» de l'historiographie américaine de la Russie, mais aussi, plus simplement deux amis, Richard Pipes (1923-2018) et Marc Raeff (1923-2008). Ce travail enrichit considérablement notre connaissance de l'historiographie américaine de la Russie. Rappelons qu'en France, le plus connu des historiens de « l'école américaine » de la Russie fut pendant longtemps Martin Malia (1924-2004)4, Pipes et Raeff n'ayant droit qu'à une diffusion confidentielle, ce qui pourrait étonner, Malia ayant le moins publié des trois auteurs. Pipes, auteur de vingt ouvrages, est désormais bien mieux connu ${ }^{5}$. De son côté, Raeff, auteur de sept monographies dont aucune n'a été traduite chez nous (!), a bénéficié d'une circulation plus informelle, mais intense, grâce au réseau de l'EHESS et l'appui d'amis influents comme Alain Besançon ${ }^{6}$.

Dans une très riche introduction, Jonathan Daly raconte les « vies parallèles » de Pipes et Raeff, analyse leurs origines sociales et intellectuelles, leurs héritages respectifs et leur 
correspondance. Tous deux sont juifs et réfugiés. Pipes, né en Pologne, fuit son pays après l'invasion germano-soviétique et arrive aux États-Unis en 1940 avec ses parents, après une aventure digne d'un roman. Raeff, né en URSS, vient aux États-Unis l'année suivante, après un passage par la France, ce qui explique sa très bonne connaissance de notre langue (il était quadrilingue). En 1946, tous deux commencent leurs études (graduate studies) à Harvard, suivent le séminaire de Michael Karpovich (1888-1959), émigré russe élève de Vassili Klioutchevski et fondateur des études russes aux États-Unis qui a formé, outre Pipes et Raeff, trois autres historiens " piliers »: Martin Malia déjà mentionné, Nicholas Riasanovsky et Leopold Haimson ${ }^{7}$. Tous deux soutiennent leur thèse en 1950, mais c'est Pipes qui, en décembre 1957, obtient le précieux sésame d'un poste de titulaire à Harvard, succédant à Karpovich (qui, on le sait, aurait préféré Malia). Raeff multiplie, lui, les postes de contractuel, avant de décrocher un poste de titulaire à Columbia en 1961.

3 Ce qui rapproche initialement Pipes et Raeff, outre le fait d'être des historiens nés à dixsept jours d'intervalle et d'être tous deux des Américains d'origine juive est-européenne, c'est leur goût pour l'histoire intellectuelle, l'intelligentsia («The importance of this group is enormous, not only for the study of Russian history but for the understanding of much of modern non-Western history in general », lettre de Pipes du 4 juin 1955). Goût inspiré par leur maître, Michael Karpovich, le patriarche des études américaines de la Russie, un homme qui a peu écrit, mais a influencé des centaines de chercheurs par son ouverture d'esprit ${ }^{8}$. Pipes et Raeff nourrissent une hostilité farouche au communisme soviétique, perçu comme un totalitarisme comparable au nazisme ; tous deux constatent la faiblesse de la société civile, ne croient pas vraiment à l'avènement prochain d'une vraie démocratie dans ce pays; tous deux rejettent l'approche "révisioniste» d'historiens américains de l'URSS comme Stephen Cohen ou Jerry Hough. Dans le même temps, beaucoup de choses les séparent, à commencer par leurs origines sociales, les orientations politiques et religieuses de leurs parents - ceux de Raeff ont été des Juifs socialistes et athées, ceux de Pipes, plus conservateurs et (modérément) pratiquants.

Surtout, Raeff et Pipes divergent quant à leur rapport à l'acte épistolaire. Raeff aime les longues discussions, cultive le plaisir épistolaire pur, à la manière de l'épistolier du $\mathrm{XIX}^{\mathrm{e}}$ siècle, aime, au cours de sa période de jeunesse, écrire de longues lettres où il s'épanche. Pipes, de son côté, se montre bien plus laconique, plus pudique, pensant que les lettres sont une activité obligée, mais, au fond, une perte de temps ( an evil made necessary by distance», 5 mai 1956). Cette différence renvoie à une divergence plus profonde, dans la définition de l'amitié en tant que pratique. Si Pipes a pu dire que Raeff a été son «meilleur ami », il n'en demeure pas moins qu'il s'est le plus souvent montré distant dans ses lettres, évitant les familiarités et les descriptions trop poussées de son quotidien. Daly y perçoit la trace d'une conception «américaine » de l'amitié, avec de très nombreuses connaissances appelées « amis ", à l'inverse de Raeff, qui cultive un petit groupe d'amis avec lesquels il cherche une relation « exclusive ». Autant dire que Raeff a bien plus écrit à Pipes que l'inverse. Et c'est aussi Raeff qui a le plus souvent «l'initiative » dans cette correspondance.

5 L'ouvrage publié par Daly contient 159 lettres, échangées entre le 26 septembre 1948 et le 11 décembre 2007, soit près de soixante ans de correspondance ${ }^{9}$. La période la plus riche est incontestablement celle des années cinquante (chapitre 1, The Early Years et surtout le chapitre 2, Uncertainty and Travel: Seattle, Berkeley, Paris), lorsque les deux anciens doctorants de Karpovich enseignent d'abord comme contractuels, puis débutent une 
carrière de titulaires, l'un à Harvard, l'autre à Columbia. La fin des années 1950 est aussi, il convient de le souligner, une période faste pour les études russes en Occident, de regain d'intérêt considérable pour la Russie soviétique aux États-Unis et dans le monde, en bonne partie lié à la possibilité offerte aux historiens de s'y rendre à nouveau pour leurs travaux de recherche. Il est amusant de constater que le déclin de la « russophilie » en Occident et le regain de tensions entre les superpuissances coïncident avec le refroidissement des relations entre Pipes et Raeff : la correspondance cesse en 1959, avant de reprendre, quatorze ans plus tard, avec une tonalité plus mesurée, formelle et distante (chapitre 3, Mature Friendship).

6 Si de nombreuses thématiques peuvent être distinguées au cours de leur principale période de correspondance, c'est surtout la question de l'origine de cette rupture dans une amitié solide qui nous préoccupera ici. On peut en chercher la racine dans des attitudes divergentes face au lot commun des jeunes docteurs en sciences humaines, l'incertitude quant à son avenir et les tentatives de décrocher le précieux sésame, le poste de titulaire (tenure). Le plus anxieux (et complexé) est assurément Raeff. Pendant longtemps, il se plaint souvent de sa surcharge de travail, de sa mémoire qu'il dit " défaillante » (5 janvier 1952), de son incertitude quant à son avenir - il passe plusieurs années à l'université de Worcester. Pipes, qui enseigne pendant plusieurs années à Harvard en contractuel, est aussi aux antipodes d'un carriériste. Mais il se montre bien plus serein que son ami, conséquence sans doute de sa «foi dans la Providence », lui qui est parvenu à quitter l'Europe in extremis en 1940, mais aussi de l'aide financière apportée par sa famille et sa belle-famille. Pour Pipes, la priorité, c'est son livre et non un poste quelconque, d'où par exemple son refus de travailler à l'université de l'Indiana en 1952-1953.

De fait, les deux hommes discutent bien évidemment aussi de leurs projets respectifs, de leurs méthodes et sources, de leurs approches de l'histoire. Si Raeff assure Pipes de son soutien et de son amitié, ses critiques sont souvent franches et brutales. Il l'est d'ailleurs avec bien d'autres historiens de la Russie comme Martin Malia ( Malia ne va certainement pas donner satisfaction", 23 septembre 1952) ou Nicholas Riasanovsky ("S'il ne change pas sa thèse, son livre sera médiocre », 28 octobre 1952). Pour ce qui est de Pipes, quand il donne son avis, tardivement, sur son premier livre, The Formation of the Soviet Union (1954), il confesse, " parce que l'amitié présuppose l'honnêteté », qu'il n'a pas eu la force de terminer le livre, qui sent trop « l'histoire événementielle [en français] » à son goût (3 mars 1956). La critique (une parmi d'autres), assez mesquine, a dû blesser Pipes qui avait reçu pour sa première monographie les éloges de son maître, Karpovich. Mais la goutte qui fait déborder le vase est la lettre très blessante de Raeff du 3 février 1958 faisant suite à la nomination de son ami au poste de titulaire à Harvard (« la chaire Karpovich »), en décembre 1957. Raeff, qui ne croit alors pas dans ses chances de trouver un poste stable, déprime et, comme c'est souvent le cas, cherche des raisons " rassurantes » à ce qu'il perçoit comme son échec. Convaincu que « les dés sont pipés » (sans mauvais jeu de mots), il accuse Pipes d'avoir «manœuvré » pour décrocher son poste. Un vieux complexe d'infériorité doublé d'anciennes rancunes remonte à la surface : Raeff en veut depuis longtemps à Pipes de se montrer avare en compliments à son égard, et il lui en veut aussi pour la relative sécheresse de sa correspondance. Pipes lui répond en disant de ne pas confondre franchise et mesquinerie et se défend d'avoir « joué à l'apparatchik» pour vaincre Malia: après tout, c'est le grand nombre de ses 
publications, comparé à ce dernier, qui lui a permis d'obtenir gain de cause. Et donc, six lettres après ce courrier de "félicitations ", la correspondance s'interrompt.

Les raisons de cette rupture, comme le montre bien Daly, sont complexes. On peut considérer que le séjour de Raeff et Pipes à Rome, pour le Congrès des Sciences historiques, en septembre 1955, marque l'apogée de leur amitié. Pour comprendre l'éloignement progressif des deux amis, il faut tenir compte de divergences dans les tempéraments des deux hommes, notamment dans la politique : plus d'une fois, Raeff critique Eisenhower, qu'il qualifie d'« idiot de la Maison-Blanche » (cf. lettre du 6 août 1957), alors que Pipes, également démocrate à cette époque, reste bien plus mesuré dans ses propos.

Raeff est surtout agacé par ce qu'il perçoit comme une incapacité de Pipes à « lâcher prise » dans une relation épistolaire, à se laisser entraîner dans une discussion qui ne soit pas un simple duel (Raeff, 9 avril 1956). Il est aussi agacé par ce qu'il perçoit comme de la condescendance. Dans une lettre datée du 6 mai 1956, inhabituellement longue, Pipes conseille à Raeff, pour décrocher un bon poste à l'université, de publier " un bon livre ", conseil que ce dernier prend mal, y voyant la preuve d'un « manque de tact évident » de la part de son ami (9 mai 1956) ${ }^{10}$. De son côté, Pipes a conscience d'avoir pu heurter son ami par son paternalisme et sa froideur et avait déjà tenté d'y remédier (" I have always treated you badly, and I shall try to make amends now », 4 juin $\left.1955^{11}\right)$. On notera tout particulièrement sa suggestion à Raeff de donner des séminaires à la future EHESS, alors $\mathrm{VI}^{\text {e }}$ Section de l'École pratique des hautes études ( You would be an excellent man to give such a course ", 19 octobre 1956), idée qui, des années plus tard (c'est Martin Malia qui est le premier à donner des cours à Paris), permettra à Raeff de se faire enfin connaître du public français avec Comprendre l'Ancien Régime russe.

Quatorze ans passent. En 1973, Pipes perd son père; Raeff lui écrit pour présenter ses condoléances - il est lui-même orphelin de père depuis longtemps. Touché, Pipes lui répond et la correspondance reprend, sur un rythme moins intense que par le passé cependant ${ }^{12}$. Pipes est alors une célébrité : son livre La Russie de l'Ancien Régime rencontre un succès considérable, son cours sur la révolution russe, dispensé à partir de février 1976, draine les foules, il est remarqué par des hommes politiques comme le sénateur démocrate Henry Jackson ( «I have become a kind of 'celebrity' on strategic nuclear matters and can hardly find time to sit down and do serious history ", 31 juillet 1977) et, en 1981, il rejoint l'équipe de Reagan en tant qu'expert ès URSS au sein du National Security Council, pour deux ans. Raeff lui marque sa déférence, voyant d'un bon œil la présence de son ami au sein de l'administration Reagan, et ce, même s'il est démocrate (il se montrera en revanche bien plus circonspect avec le projet de réformes de Gorbačev). Pipes lui envoie ses publications, Raeff lui donne son avis, le plus souvent élogieux, le félicitant pour sa biographie de Pierre Struve (« and you write so well and vividly ", 16 mars 1980), non sans petites piques ici ou là. En retour, Pipes encourage Raeff à écrire son histoire de l'émigration comme il ne l'a jamais fait («I very much hope that you undertake a history of the Russian emigration, which is badly needed », 2 avril 1980). En dépit de tentatives de semer la discorde entre les deux hommes en faisant passer Comprendre l'Ancien Régime russe de Raeff, à tort, pour "l'antithèse " de La Russie de l'Ancien Régime ${ }^{13}$, l'amitié persiste, avec une complicité parfois étonnante. Raeff prend sa retraite en 1994, Pipes en 1996, et les deux hommes la vivent très différemment : le premier, qui n'a plus la force de se rendre dans les archives russes, abandonne toute 
recherche et jouit de sa "liberté retrouvée », le second continue de voyager, de publier et déprime s'il ne travaille pas quatre à cinq heures par jour.

11 La correspondance entre Richard Pipes et Marc Raeff passionnera sans doute ceux qui sont curieux de l'historiographie de la Russie outre-Atlantique dans sa tendance libérale, anticommuniste. Les états d'âme et la franchise de Raeff sont les aspects les plus intéressants de la publication; on en apprend moins sur Pipes, pour ceux qui connaissent ses mémoires ${ }^{14}$. On pourrait regretter les silences, entre 1958 et 1973 (les réactions des deux hommes aux tourments des universités américaines dans les années soixante auraient été intéressantes), le hiatus inexpliqué entre octobre 1984 et septembre 1987, la discrétion des deux hommes sur leurs étudiants (contrairement à une légende noire, Pipes a beaucoup soutenu ses anciens doctorants et entretenu une correspondance suivie avec beaucoup d'entre eux). En dépit de ces réserves, la correspondance donne un bon aperçu du "tout petit monde» des universités américaines. L'universitaire français pourrait y trouver matière à réflexion et à comparaison, en dépit des différences, et elles sont immenses, entre les deux systèmes d'enseignement supérieur et quelles que soient, par ailleurs, ses orientations historiographiques.

\section{NOTES}

1. Bertrand Müller, éd., Marc Bloch, Lucien Febvre: Correspondance, Fayard, 2003-2004 (trois volumes). François Furet et Ernst Nolte, Fascisme et communisme, Plon, 1998.

2. Voir ses deux ouvrages Autocracy under Siege : Security Police and Opposition in Russia, 1866-1905, Northern Illinois University Press, 1998 et The Watchful State : Security Police and Opposition in Russia, 1906-1917, Northern Illinois University Press, 2004.

3. Voir son article programmatique «The Unknown Richard Pipes : On the Craft and Philosophy of History ", Ab Imperio, 1 (2019), p. 237-265.

4. Voir ses ouvrages Comprendre la Révolution russe (Seuil, 1980), La tragédie soviétique (1995), L'Occident et l'énigme russe (2003), et le posthume Histoire des révolutions (Seuil, 2010).

5. Pour Pipes, il faut attendre 1993 pour la publication de sa Révolution russe (PUF, 1993), rééditée récemment aux éditions Perrin (Les révolutions russes, 2017). Puis, il faut attendre ma traduction de Russia under the Old Regime (Histoire de la Russie des tsars, Perrin, 2013, édition Tempus en 2017). Au cours de la même époque sont sortis, aux éditions De Fallois, L'Affaire Degaev (2011) et Les Trois pourquoi de la révolution russe (2013). Parmi les œuvres inédites en France, sa biographie en deux volumes de Pierre Struve, le «premier repenti du marxisme » (1970), et Russia under the Bolshevik Regime (1995), le troisième volume de sa "trilogie révolutionnaire ».

6. Le seul ouvrage en français fut longtemps Comprendre l'Ancien Régime russe (Seuil, 1982), issu de séminaires donnés à l'EHESS en 1980. Après la fin de l'URSS, paraît Politique et culture en Russie, $X V I I I^{e}-X X^{e}$ siècles (EHESS, 1996). Les travaux qui auraient mérité être traduits: The Well-Ordered Police State: Social and Institutional Change Through Law in the Germanies and Russia, 1600-1800 (1983), dont les thèses sont énoncées dans l'ouvrage de 1982 susmentionné ; et son dernier livre, une histoire de l'émigration russe (Russia Abroad : A Cultural History of the Russian Emigration, 1919-1939, 1990). 
7. Jonathan Daly, «The Pleiade : Five Scholars who Founded Russian Historical Studies in the United States ", Kritika, 18, 4 (2017), p. 785-826.

8. Norman G.O. Pereira, « The Thought and Teachings of Michael Karpovich », Russian History, 36 (2009), p. 254-277.

9. De nombreuses lettres sont manquantes, et notamment celle de Raeff, datée d'avril 1973, qui relance la relation épistolaire après un hiatus de quatorze ans.

10. Le premier livre de Raeff, la biographie de Speranski, verra le jour en 1957.

11. Cette lettre, où Pipes répond aux critiques de Raeff sur son article "Max Weber and Russia » (World Politics, 5 , 3 (1955)), éclaire l'origine de l'ouvrage qui fera la gloire de Pipes, La Russie de l'Ancien Régime.

12. La lettre de condoléances de Raeff est absente, tout comme d'autres courriers.

13. Voir la lettre du 4 octobre 1984 de Pipes, qui fait référence à la recension d'Andreas Kappeler un célèbre historien suisse. En France, Alain Besançon perpétue ce malentendu, contre l'avis de Raeff - voir mon introduction à ma traduction du livre, mentionnée plus haut.

14. Richard Pipes, Vixi, Memoirs of a Non Belonger, Yale University Press, 2003.

\section{AUTEURS}

\section{ANDREÏ KOZOVOÏ}

Université de Lille 